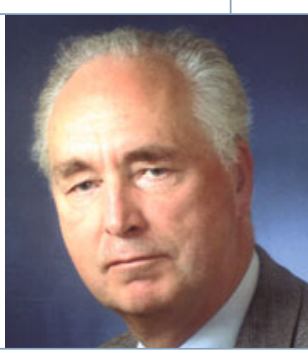

Hans-Dieter Klenk

\title{
Manipulation am Vogelgrippevirus: Segen oder Fluch?
}

Seit dem ersten Auftreten des aviären Influenza-A-Virus vom Subtyp H5N1 im Jahre 1997 musste man befürchten, dass aus dem hochpathogenen „Vogelgrippevirus“ ein Erreger mit hoher Übertragbarkeit beim Menschen entstehen könnte. Mit dem Pandemieausbruch von 2009, der entgegen aller Voraussagen durch ein H1N1-Virus („Schweinegrippe") verursacht wurde, verschwanden die H5N1-Erreger aus dem Blickfeld. Jetzt gewinnen sie neue Aktualität, da offenbar im Labor H5N1-Viren hergestellt wurden, die Eigenschaften eines Pandemieerregers haben. So berichtete Ron Fouchier, Erasmus Medical Center, Rotterdam, im vergangenen Herbst über Experimente, in denen H5N1-Viren durch gentechnische Manipulation und klassische Adaptionsverfahren so verändert wurden, dass sie bei Frettchen auf dem Luftwege übertragen wurden, ohne dabei ihre hohe Pathogenität zu verlieren. Erstaunlicherweise reichten offenbar wenige Mutationen im Virusgenom für den Wirtswechsel aus. Ähnliches zeigten die Arbeitsgruppen von Yoshirio Kawaoka, School of Veterinary Medicine, Madison, USA.

Diese Untersuchungen sollten zentrale Probleme der Influenzavirusforschung klären, nämlich die zugrunde liegenden Mechanismen der Pathogenität, der Wirtsspezifität und der Übertragbarkeit der Viren. Darüber hinaus waren wichtige Informationen für die Überwachung der Influenza bei Mensch und Tier zu erwarten. Sollten nämlich die in diesen Studien beobachteten Mutationen in der Natur auftreten - nichts spricht dagegen -, wäre größte Wachsamkeit geboten. Trotz der großen wissenschaftlichen Bedeutung dieser Arbeiten darf nicht übersehen werden, dass sie für bioterroristische und ähnliche Zwecke missbraucht werden könnten. Da Pathogenität und Übertragbarkeit der Influenzaviren sich bei Frettchen und Mensch ähneln, ist nicht auszuschließen, dass von den experi- mentell veränderten H5N1-Viren eine erhebliche Gefahr für den Menschen ausgeht.

Diese Dual-Use-Problematik war in den vergangenen Wochen Gegenstand intensiver Diskussionen in der Fachwelt und schließlich in der Öffentlichkeit. Auch das National Science Advisory Board for Biosecurity (NSABB) der USA befasste sich damit und verhinderte die Veröffentlichung der bei Science und Nature eingereichten Manuskripte. Nun werden Maßnahmen debattiert, die im aktuellen und in ähnlichen zukünftigen Fällen angewendet werden könnten. Auf drei Maßnahmen wird hier kurz eingegangen: (1) Arbeiten mit H5N1Viren sollen nur in Laboratorien mit der höchsten Sicherheitsstufe durchgeführt werden, also Stufe 4, statt wie bislang 3. Die Konsequenz wäre eine massive Einschränkung der Influenzaforschung weltweit, da nur ganz wenige der auf diesem Gebiet tätigen Gruppen über BSL4-Laboratorien verfügen. (2) Arbeiten sensiblen Inhalts sollen nur unter Ausschluss der experimentellen Daten veröffentlicht werden. Diese Maßnahme würde eindeutig gegen gute wissenschaftliche Praxis verstoßen, da dann jegliche Überprüfung der erhobenen Befunde und ihre Beurteilung im Kontext mit anderen Untersuchungen unmöglich wären. Auch würden so Spekulationen aller Art und einer unangemessenen Dämonisierung Tür und Tor geöffnet. (3) Am weitesten geht die Forderung, nach der Arbeiten, die zur Pathogenitätssteigerung eines Erregers führen, grundsätzlich verboten werden sollen. Gefährliche Erreger sind aber nur dann erfolgreich zu bekämpfen, wenn wir verstehen, worauf deren Pathogenität beruht. Daher müssen wir die Pathogenität experimentell verändern, auch wenn dies mit einem erhöhten Gefahrenrisiko einhergeht. Aus wissenschaftlicher Sicht kann also keiner dieser Forderungen zugestimmt werden.

Eine abschließende Bewertung der beiden Studien ist ohne genaue Kenntnis der Daten nicht möglich. Jedoch ist der Zugewinn an wissenschaftlicher Erkenntnis höher zu bewerten als das Risiko, das von den modifizierten Viren ausgeht. Diese Einschätzung teilen auch die Gesellschaft für Virologie sowie die Leopoldina. Leopoldina-Präsident Prof. Dr. Jörg Hacker betont, dass die aktuellen Forschungsergebnisse „für die Grundlagenforschung und die Entwicklung neuer Medikamente und Impfstoffe gegen die Vogelgrippe Bedeutung haben“. Letztlich muss die Infektionsforschung das Pro und Kontra der Dual-Use-Problematik verantwortungsvoll gegeneinander abwägen, so wie sie das von Anfang an getan hat: Mit der Isolierung vom Milzbranderreger Bacillus anthracis begründete Robert Koch die Bakteriologie als die wahrscheinlich erfolgreichste Disziplin der Medizin. Gleichzeitig hatte man aber einen Erreger mit Biowaffenpotenzial in Händen, was später wiederholt ausgenutzt wurde. Trotzdem hat niemand Koch die Erforschung des Milzbranderregers verboten, zum Glück.

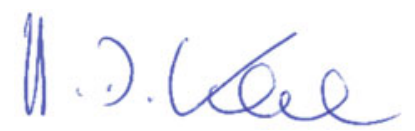

Hans Dieter Klenk, Institut für Virologie, Philipps-Universität Marburg

Korrespondenzadresse:

Prof. Dr. Hans-Dieter Klenk Philipps-Universität Marburg Institut für Virologie Hans-Meerwein-Straße 2 D-35043 Marburg Tel.: 06421-28-66191/66254 Fax: 06421-28-68962 klenk@staff.uni-marburg.de 